\title{
Drug-induced Hypersensitivity Syndrome in an ANA-negative, anti-dsDNA-negative Systemic Lupus Erythematosus Patient
}

\author{
Ann Meredith U. Garcia, ${ }^{1}$ Geraldine Z. Racaza, ${ }^{1}$ Paolo S. Macasaet ${ }^{2}$ and Agnes D. Mejia ${ }^{1}$ \\ ${ }^{1}$ Department of Medicine, College of Medicine and Philippine General Hospital, University of the Philippines Manila \\ ${ }^{2}$ Department of Pathology, College of Medicine, University of the Philippines Manila
}

\begin{abstract}
A 50-year-old female presented with rash and multi-organ toxicity after intake of several antibiotics. This unmasked an elderly-onset, non-drug-induced seronegative systemic lupus erythematosus (SLE) which was confirmed by lupus band test (LBT) and skin biopsy staining. This patient presented with the rare combination of drug-induced hypersensitivity syndrome (DIHS) and SLE.
\end{abstract}

Key Words: drug-induced hypersensitivity syndrome, systemic lupus erythematosus, lupus band test

\section{Case Presentation}

A previously healthy 50-year-old female presented at our emergency room (ER) with decreased sensorium.

Six weeks prior to admission (PTA), she experienced fever, dysuria, and bipedal edema which prompted consult at a local hospital. Urinalysis showed pyuria [white blood cell (WBC) 18-25/high-power field (HPF)] with bacteriuria and proteinuria $(+3)$. Complete blood count $(\mathrm{CBC})$ revealed leukocytosis (WBC $13.8 \times 109 / \mathrm{L}$ ) with $85 \%$ neutrophil predominance and a hemoglobin $(\mathrm{Hb})$ level of $165 \mathrm{~g} / \mathrm{L}$. She was treated for urinary tract infection with cefuroxime. She was also given hydrochlorothiazide (HCTZ) and prednisone, presumably for the edema and proteinuria. Three days later, she developed erythematous, target-like plaques starting on the extremities then involving the trunk. She also had oral ulcers and occasional joint and low back pain.

The patient consulted another physician who shifted her antibiotic to ciprofloxacin for 2 days, then to co-amoxiclav. After another 2 days, the patient discontinued the antibiotics and instead took herbal medications (i.e., reishi gano, ganocelium, spirulina, morinzhi, ganozhi) prescribed by an iridologist. Her skin lesions progressed, prompting consult

Presented at the Philippine Rheumatology Association 20th Annual Meeting, February 7-9, 2013, Taal Vista Hotel, Tagaytay City; 1st Asian Lupus Summit, November 29-30, 2012, EDSA Shangri-La Hotel, Mandaluyong City; and Philippine College of Physicians 42nd Annual Convention Free

Communication Sessions, May 6-9, 2012, SMX Convention Center, Pasay City.

Corresponding author: Agnes D. Mejia, MD

Department of Medicine

Philippine General Hospital

University of the Philippines Manila

Taft Avenue, Ermita, Manila 1000 Philippines

Telephone: +632 5548400 local 2200/2206

Telefax: +6325264372

Email: agnesmejiamd@yahoo.com at the Dermatology outpatient clinic of PGH. The initial clinical impression was erythema multiforme major. A 4-mm skin punch biopsy was performed and she was given antihistamines.

On follow-up, repeat urinalysis showed decreased pyuria (WBC 4-8/HPF) with persistent bacteriuria and proteinuria $(+1)$, as well as fine granular casts $(1-3 / \mathrm{HPF})$. She had the following pertinent blood test results:

$\begin{array}{ll}\text { Hemoglobin }(\mathrm{Hb}) & 110 \mathrm{~g} / \mathrm{L} \downarrow \\ \text { WBC } & 9.0 \times 10^{9} / \mathrm{L} \\ \text { Creatinine } & 93 \mu \mathrm{mol} / \mathrm{L} \\ \text { Aspartate aminotransferase (AST) } & 63 \mathrm{U} / \mathrm{L} \uparrow \\ \text { Alanine aminotransferase (ALT) } & 49 \mathrm{U} / \mathrm{L} \uparrow \\ \text { Sodium } & 123 \mathrm{mmol} / \mathrm{L} \downarrow \\ \text { Albumin } & 23 \mathrm{~g} / \mathrm{L} \downarrow \\ \text { Erythrocyte sedimentation rate (ESR) } & 60 \mathrm{~mm} / \mathrm{hr} \uparrow\end{array}$

The skin biopsy revealed superficial and deep perivascular dermatitis with interface changes consistent with drug reaction (Figure 1). She was given ebastine plus low-dose betamethasone for one week and was referred to the Internal Medicine department. However, she did not seek consult.

The patient subsequently developed pallor and oliguria with worsening generalized weakness, malaise, and anorexia until she was noted to have altered sensorium 1 day PTA.

At the ER, the patient was received aphasic without focal neurologic deficits. She was pale and tachycardic, with persistent generalized target-like plaques and bipedal edema. She was leukopenic (WBC 1.9 x 109/L) with neutropenia [absolute neutrophil count 1,292/ $\mu \mathrm{L}$ ] and had severe normocytic, normochromic anemia ( $\mathrm{Hb} 58 \mathrm{~g} / \mathrm{L})$. She also had a low reticulocyte production index, negative Coombs' tests, and slightly elevated lactate dehydrogenase (LDH) (575 U/L). She was transfused with packed red blood cells (RBCs) with subsequent improvement in sensorium. Repeat CBC showed a $\mathrm{Hb}$ level of $97 \mathrm{~g} / \mathrm{L}$ with persistent leukopenia (WBC $\left.2.1 \times 10^{9} / \mathrm{L}\right)$ and new-onset thrombocytopenia (platelet count $105 \times 10^{9} / \mathrm{L}$ ). Prednisone was started at $1 \mathrm{mg} / \mathrm{kg} /$ day for a possible drug-induced versus autoimmune condition, particularly SLE. Her blood counts temporarily stabilized despite persistent pancytopenia. 


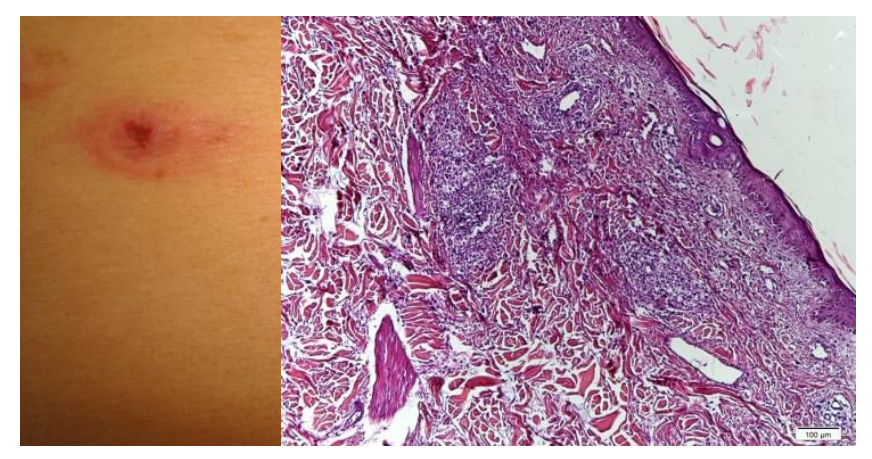

Figure 1. H\&E stain (right) of a target-like plaque (left), showing superficial and deep perivascular dermatitis with interface changes consistent with drug reaction.

She had episodes of congestion associated with an S3 gallop despite a negative fluid balance. Electrocardiograms were nonspecific with normal troponin I levels. Transthoracic echocardiogram showed concentric left ventricular hypertrophy with multisegmental wall motion abnormalities and a depressed ejection fraction (EF) of $31 \%$. Thus, myocarditis was considered.

The patient also developed pneumonia on the $4^{\text {th }}$ hospital day. Piperacillin-tazobactam was started but she continued to have intermittent fever despite initial clinical improvement and persistently negative cultures.

On further work-up, antinuclear antibody (ANA) and anti-double-stranded DNA (anti-dsDNA) were both negative. Thus, steroids were tapered on the $14^{\text {th }}$ hospital day. DIHS from HCTZ or cefuroxime with possible macrophage activation syndrome (MAS) became the major consideration, supported by markedly elevated serum ferritin (>2000 ng/mL), fasting triglycerides (449.56 mg/dL), and $\operatorname{LDH}(3,332 \mathrm{U} / \mathrm{L})$.

Upon steroid tapering, worsening of anemia and thrombocytopenia ensued despite immediate resumption of high-dose steroid therapy. The patient also developed newonset lymphadenopathies, as well as new erythematous plaques which were biopsied. Lupus band test (LBT) was performed.

The patient eventually started to exhibit waxing and waning sensorium. The plain cranial computed tomography scan showed only cerebrocerebellar atrophy. On the $16^{\text {th }}$ hospital day, she went into shock and was admitted in the intensive care unit. Her initial clinical picture was most consistent with hypovolemia and a probable cardiogenic component from myocarditis. Repeat cultures were still negative and fever lysis was observed during and after a naproxen test.

There was a further drop in platelet count $\left(56 \times 10^{9} / \mathrm{L}\right.$ to a nadir of $\left.21 \times 10^{9} / \mathrm{L}\right)$ and $\mathrm{Hb}(48 \mathrm{~g} / \mathrm{L})$. Upper gastrointestinal bleeding and pulmonary hemorrhage followed, prompting transfusion of packed RBCs and platelet concentrates. The patient was then intubated due to pulmonary edema with persistent hypoxemia. Careful diuresis was performed with maintenance of inotropic support. Acute respiratory distress syndrome was also considered and she was maintained on low tidal volume ventilation. She had refractory shock accompanied by progressive acute renal failure, culminating in coma and cardiac arrest on the $19^{\text {th }}$ hospital day.

Skin biopsy results revealed a positive lupus band test with continuous thick granular deposition of immunoglobulin $\mathrm{M}$ (IgM) along the basement membrane zone (BMZ) (Figure 2) and a positive Alcian blue stain for mucin (Figure 3). Both findings are consistent with lupus erythematosus with vasculitis.

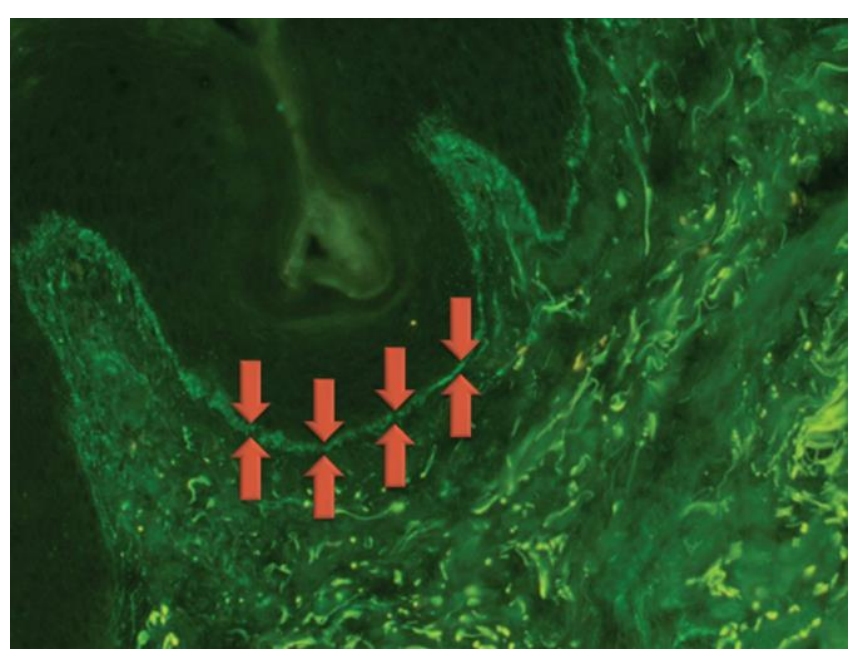

Figure 2. Direct immunofluorescence showing a positive lupus band test with continuous thick granular deposition of IgM along the basement membrane zone (actual specimen not documented; image courtesy of Reich et $\mathrm{al}^{17}$ ).

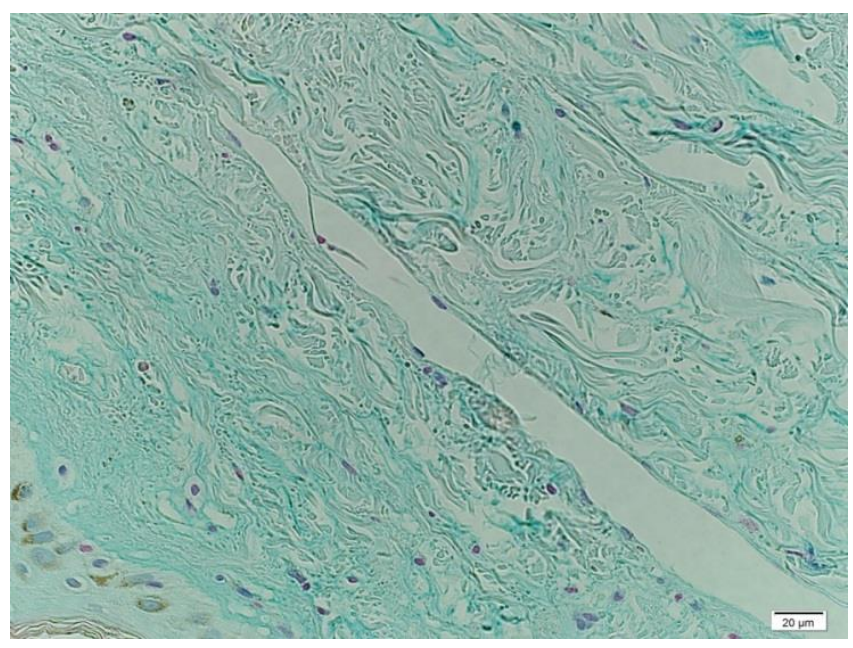

Figure 3. Positive Alcian blue stain indicating the presence of dermal mucin. 
A postmortem study was performed revealing pulmonary hemorrhage as the probable cause of death. Bone marrow morphologic features were supportive of myelodysplastic syndrome (MDS) with World Health Organization classification of refractory cytopenia with multilineage dysplasia. Other significant abnormal findings included pulmonary congestion, multiple transmural ulcers and serosal adhesions in the jejunum and ileum, pericardial effusion, and ascites. The heart was unremarkable, as well as the brain and kidneys.

\section{Discussion}

We present a case in which the temporal relationship of signs and symptoms to drug intake and initial skin biopsy findings pointed to a drug reaction. However, with accompanying multisystemic features, an underlying autoimmune process was also considered. This paper will discuss DIHS, SLE, drug-induced lupus erythematosus (DILE), and coexistent DIHS and SLE.

\section{Drug-induced Hypersensitivity Syndrome}

DIHS is a life-threatening, multisystemic drug reaction with a highly variable clinical picture. ${ }^{1}$ It is also known as drug rash with eosinophilia and systemic symptoms (DRESS) syndrome. However, the term DIHS is now preferred because eosinophilia is seen at most in only 60$70 \% .^{2}$ It has several unique features, including a delayed onset of 1-8 weeks, paradoxical deterioration of clinical symptoms after drug withdrawal, and unexplained crossreactivity to unrelated drugs. ${ }^{1,2}$ It can also present with elevated serum LDH, ferritin, and triglycerides, particularly when accompanied by hemophagocytosis or MAS. ${ }^{3}$

DIHS can be a diagnostic dilemma as there is no universal set of diagnostic criteria. ${ }^{4} \mathrm{~A}$ recent case series by Um et al. utilized the following criteria: 1) acute cutaneous eruption; 2$)$ fever $\left(>38^{\circ} \mathrm{C}\right.$ in a patient with a history of taking a specific drug); and 3) at least one internal organ abnormality (i.e., lymphadenopathy at a minimum of 2 sites, hepatitis, nephritis, pneumonitis, carditis, thyroiditis, hematological abnormalities). ${ }^{5}$

\section{Systemic Lupus Erythematosus}

SLE is a heterogeneous chronic autoimmune disorder characterized by the presence of autoantibodies, complement consumption, and circulating immune complexes resulting in inflammatory damage to multiple organ systems. ${ }^{6,7}$ It is a clinical diagnosis supported by laboratory investigations based on the 1997 update of the 1982 American College of Rheumatology (ACR) revised criteria. ${ }^{8,9}$

ANA is an important screening test for all types of SLE, ${ }^{6}$ with sensitivity over $95 \%$ and specificity of $57 \%$ using immunofluorescence, the current gold standard. ${ }^{10}$ However, negative serological tests do not always exclude SLE as these tests may become positive at a later period. Current expert opinion suggests an incidence of $<2 \%$ for true ANA-negative SLE. ${ }^{11}$

\section{Drug-induced Lupus Erythematosus}

DILE should be considered in patients who develop lupus-like manifestations (i.e., arthralgias, myalgias, fever, pleurisy, pericarditis) while taking a drug, accompanied by a positive ANA and anti-histone antibodies, followed by prompt improvement upon drug discontinuation. ${ }^{12} \mathrm{HCTZ}$, a sulfonamide, is among the most common culprit drugs. ${ }^{13}$

Histopathology and IF findings are very similar to those of idiopathic SLE. However, skin involvement is generally less frequent in DILE. Severe cytopenias are unlikely, while CNS and renal involvement is usually absent. ${ }^{12}$ DILE usually develops only after months to years of drug intake. Irreversibility upon discontinuation of the drug is an indication that the drug unmasked underlying SLE, ${ }^{14}$ making DILE less likely in our patient.

\section{DIHS and SLE Overlap}

Viral reactivation and immune dysfunction involving activated regulatory $\mathrm{T}$ cells play an important role in DIHS. Several autoimmune diseases have been reported to occur as sequelae of DIHS even up to several years after resolution ${ }^{2}$ of clinical conditions. SLE presenting with KikuchiFujimoto's disease as a long-term sequela of DIHS has been documented once, but this occurred 4 years after the resolution of DIHS. ${ }^{15}$ Prior to this paper, there has been no report of the simultaneous occurrence of DIHS and SLE.

The cutaneous lesions were critical to the diagnosis of this patient. Erythema multiforme is among the nonspecific skin manifestations of lupus. ${ }^{16}$ In SLE, vacuolar degeneration of the basal layer with thickened subepidermal basement membrane accompanied by perivascular lymphocytic infiltrates are characteristic, ${ }^{17}$ as well as dermal mucinosis confirmed by the Alcian blue stain. ${ }^{13}$ Furthermore, Ig and complement deposition in the skin of patients with lupus is seen as a linear band at the basement membrane zone by direct IF (i.e., the lupus band). Our patient's skin biopsy was positive for the Alcian blue stain for mucin and the lupus band test. LBT is a very sensitive (95\%) and specific (87\%) test for lupus, comparable to other laboratory variables such as ANA. ${ }^{7}$ It appears to be most useful in diagnosing SLE in patients with inconclusive clinical and serological profiles but whose conditions suggest such diagnosis. ${ }^{17}$ This was demonstrated in a previous report of a patient who fulfilled only 3 (polyserositis, thrombocytopenia, arthritis) out of the 11 ACR criteria accompanied by atypical skin lesions and a negative ANA but was eventually diagnosed with SLE based on a positive LBT. ${ }^{18}$ Interestingly, the most common skin biopsy findings in DIHS are quite similar to those in SLE, including superficial perivascular lymphocytic infiltrates, interface dermatitis, and edema and keratinocyte 
necrosis in the epidermis..$^{3,19,20}$ However, IF will be rarely positive in drug reaction. ${ }^{13}$

Another prominent aspect of this case is the hematologic involvement. Autoimmune-mediated cell destruction in peripheral blood is the most frequent cause of cytopenias in $\mathrm{SLE}^{21}$ but reversible bone marrow dysplasia can also be observed. ${ }^{22}$ Reactive hemophagocytosis has also been reported, ${ }^{23,24}$ manifested by fever, hepatosplenomegaly, lymphadenopathy, and variable cytopenias associated with elevated serum LDH, ferritin, triglycerides, and transaminases. ${ }^{3}$ Although its pathognomonic feature is bone marrow hemophagocytosis, this is not always demonstrable. Thus, failure to document hemophagocytosis does not exclude the diagnosis. ${ }^{25}$ DIHS is also characterized by macrophage activation, ${ }^{3}$ explaining their overlapping features and illustrating the complex association between these disease entities.

The patient also presented with signs and symptoms of heart failure which can be brought about by myocarditis due to SLE or DIHS. In SLE myocarditis, the most common electrocardiographic features are nonspecific ST segment and $\mathrm{T}$ wave changes. Common echocardiographic findings include segmental wall motion abnormalities and decreased left ventricular EF. ${ }^{26}$ In the pre-steroid era, it was observed that approximately 50-77\% of SLE patients had evidence of myocarditis at autopsy. ${ }^{27}$ Myocarditis has also been frequently found in patients with DIHS, and it is often accompanied by normal cardiac enzymes and rare or patchy, mixed inflammatory infiltrates on histopathologic examination. ${ }^{3}$ Our patient had normal cardiac findings on autopsy. Acute myocarditis from either cause was suspected on clinical grounds.

Our patient also presented with persistent on-and-off fever despite broad-spectrum antibiotic therapy and the absence of evidence of an infectious focus. In DIHS, fever is usually high and spiking but the results of cultures are negative. ${ }^{1}$ Connective tissue diseases (CTDs) also commonly present with fever. Although rarely used now, a naproxen test was tried in this patient with a positive response. Fever lysis is suggestive of either a possible neoplastic condition or a CTD, while a negative response is suggestive of a possible infection. A local study conducted by Dy et al. showed a sensitivity of $94.6 \%$ and a specificity of $58.5 \%$ for this test, ${ }^{28}$ suggesting that our patient's fever is less likely to be infectious in etiology.

The difficulty in diagnosing this case was brought about by the overlapping features between DIHS and seronegative SLE. The patient was able to meet the previously mentioned diagnostic criteria for DIHS (acute cutaneous eruption; fever; internal organ abnormalities in the form of cytopenias, lymphadenopathies, and possible myocarditis) accompanied by elevated LDH, ferritin, and triglycerides. Since her ANA and anti-dsDNA were both negative and she fulfilled only 3 of the 11 diagnostic criteria for SLE (oral ulcers, hematologic disorder, and history of proteinuria and cellular casts), SLE was initially thought to be unlikely. However, with positive LBT and Alcian blue stain, SLE was then highly considered. SLE could have predisposed her to develop DIHS, or SLE may have been unmasked or exacerbated by DIHS. Even prior to drug intake, she already presented with bipedal edema and abnormal urinalysis findings which may have already been signs of evolving lupus. It is thus important to remember that the ACR classification criteria were originally formulated for the inclusion of patients with "a definite diagnosis of SLE" in scientific studies and were not designed for diagnostic purposes as they lack sensitivity (78\%) for recognizing patients with milder or atypical manifestations. ${ }^{19}$

Williams et al. recommended that the LBT be performed in cases of possible lupus in the absence of positive serology. However, this should always be interpreted in conjunction with clinical findings and other serological and immunopathological parameters. ${ }^{17}$

As in SLE, it is most important to the management of DIHS to have a high index of suspicion and to recognize the disease early. The mainstay of therapy is also systemic corticosteroids, $40-60 \mathrm{mg} /$ day of prednisolone or its equivalent. Steroids need to be slowly tapered over 6-8 weeks as marked deterioration of symptoms is often observed with rapid tapering or discontinuation. If symptoms continue to deteriorate, other options used successfully in small series of patients include intravenous pulse methylprednisolone, intravenous Ig, plasmapheresis, or a combination of these.

\section{References}

1. Kumari R, Timshina DK, Thappa DM. Drug hypersensitivity syndrome. Indian J Dermatol Venereol Leprol. 2011; 77(1):7-15.

2. Shiohara T, Kano Y, Takahashi R. Current concepts on the diagnosis and pathogenesis of drug-induced hypersensitivity syndrome. JMAJ. 2009; 52(5):347-52.

3. Ben m'rad M, Leclerc-Mercier S, Blanche $\mathrm{P}$, et al. Drug-induced hypersensitivity syndrome. clinical and biologic disease patterns in 24 Patients. Medicine. 2009; 88(3):131-40.

4. Sparsa A, Loustaud-Ratti V, Mousset-Hovaere M, et al. Drug-induced hypersensitivity syndrome in internal medicine: diagnostic and therapeutic traps. Eight observations. Rev Med Interne. 2000; 21(12):1052-9.

5. Um SJ, Lee SK, Kim YH, et al. Clinical features of drug-induced hypersensitivity syndrome in 38 patients. J Investig Allergol Clin Immunol. 2010; 20(7):556-62.

6. Maraina $\mathrm{CH}$, Kamaliah $\mathrm{MD}$, Ishak M. ANA negative (Ro) Lupus Erythematosus with multiple major organ involvement: a case report. Asian Pac J Allergy Immunol. 2002; 20(4):279-82.

7. Mehta V, Sarda A, Balachandran C. Lupus band test. Indian J Dermatol Venereol Leprol. 2010; 76(3):298-300.

8. Hochberg MC. Updating the American College of Rheumatology revised criteria for the classification of systemic lupus erythematosus. Arthritis Rheum. 1997; 40(9):1725.

9. Tan EM, Cohen AS, Fries JF, et al. The 1982 revised criteria for the classification of systemic lupus erythematosus. Arthritis Rheum. 1982; 25(11):1271-7. 
10. American College of Rheumatology, POSITION STATEMENT: Methodology of Testing for Antinuclear Antibodies [Online]. 2011 [cited 2012 Apr]. Available from http://www.rheumatology.org/practice/ clinical/position/ana_position_stmt.pdf.

11. Cross LS, Aslam A, Misbah SA. Antinuclear antibody-negative lupus as a distinct diagnostic entity-does it no longer exist? QJM. 2004; 97(5):303-8.

12. Antonov D, Kazandjieva J, Etugov D, Gospodinov D, Tsankov N. Druginduced lupus erythematosus. Clin Dermatol. 2004; 22(2):157-66.

13. Jamora MJJ. Diagnostic Immunopathology in 21st Century Dermatology Part II: Basement Membrane and Vascular Reactions. Dermatol Sinica. 2008; 26:191-227.

14. Borchers AT, Keen CL, Gerhswin ME. Drug-induced lupus. Ann NY Acad Sci. 2007; 1108:166-82.

15. Aota N, Hirahara K, Kano Y, Fukuoka T, Yamada A, Shiohara T. Systemic lupus erythematosus presenting with Kikuchi-Fujimoto's disease as a long-term sequela of drug-induced hypersensitivity syndrome. A possible role of Epstein-Barr virus reactivation. Dermatology. 2009; 218(3):275-7.

16. Popovic K. Cutaneous lupus erythematosus and immunoreactivity in patients with Ro/SSA autoantibodies. Stockholm: Karolinska University Press; 2007.

17. Reich A, Marcinow K, Bialynicki-Birula R. The lupus band test in systemic lupus erythematosus patients. In: Therapeutics and Clinical Risk Management, vol. 7. Dove Medical Press Ltd; 2011. pp. 27-32.

18. Boinas C, Ambar JA, Machado C, Gomes MJM. Polyserositis: a case report. Rev Port Pneumol. 1999; 5(6):603-9.
19. Shiohara T, Inaoka M, Kano Y. Drug-induced Hypersensitivity Syndrome (DIHS): a reaction induced by a complex interplay among herpesviruses and antiviral and antidrug immune responses. Allergol Int. 2006; 55(1):1-8.

20. Ang CC, Wang YS, Yoosuff EL, Tay YK. Retrospective analysis of druginduced hypersensitivity syndrome: a study of 27 patients. J Am Acad Dermatol. 2010; 63(2):219-27.

21. Lorand-Metze I, Carvalho MA, Costallat LT. Morphology of bone marrow in systemic lupus erythematosus. Pathologe. 1994; 15(5):292-6.

22. Oka Y, Kameoka J, Hirabayashi Y, et al. Reversible bone marrow dysplasia in patients with systemic lupus erythematosus. Intern Med. 2008; 47(8):737-42.

23. Wong KF, Hui PK, Chan JK, Chan YW, Ha SY. The acute lupus hemophagocytic syndrome. Ann Intern Med. 1991; 114(5):387-90.

24. Dhote R, Simon J, Papo T, et al. Reactive hemophagocytic syndrome in adult systemic disease: report of twenty-six cases and literature review. Arthritis Rheum. 2003; 49(5):633-9.

25. Medscape Reference, Macrophage Activation Syndrome [Online]. 2012 [cited 2011 Aug]. Available from http://emedicine.medscape.com/ article/1380671-workup.

26. Law WG, Thong BY, Lian TY, Kong KO, Chng HHl. Acute lupus myocarditis: clinical features and outcome of an oriental case series. Lupus. 2005; 14(10):827-31.

27. Ansari A, Larson PH. Heart disease in systemic lupus erythematosus: diagnosis and management. Tex Heart Inst J. 1985; 12(1):9-21.

28. Dy EER, Moral PGL, Victorio-Navarra STG. The Naproxen Test as a diagnostic tool in the causative differentiation of fever. Phil J Microbiol Infect Dis. 1999; 28(3):85-90. 